\title{
PRÁCTICAS EDUCATIVAS DE LOS PADRES Y PROBLEMAS DE AJUSTE EN ESTUDIANTES UNIVERSITARIOS
}

\author{
Mª de la Villa Carpio Fernández \\ Ma Cruz García Linares \\ Pedro Félix Casanova Arias \\ Mª Teresa Cerezo Rusillo \\ Departamento de Psicología, Universidad de Jaén, \\ Campus de las Lagunillas, s/n, 23071 Jaén. \\ E-mail:mvcarpio@ujaen.es \\ https://doi.org/10.17060/ijodaep.2017.n1.v2.931
}

Fecha de Recepción: 6 Febrero 2017

Fecha de Admisión: 1 Abril 2017

\section{RESUMEN}

Este estudio tiene como objetivo analizar las relaciones entre el afecto/rechazo y los tipos de disciplina que muestran tanto el padre como la madre y los problemas internalizantes y externalizantes que informan los estudiantes universitarios.

Se aplicaron la Escala de Afecto «EA» y la Escala de Normas y Exigencias «ENE» (Fuentes, Motrico y Bersabé, 1999; Bersabé, Fuentes y Motrico, 2001) en su versión para hijos (EA-H y ENEH), así como el Youth Self- Report (Achenbach (1991) mediante la propuesta de Ivanova et al., (2007) quienes agrupan los ítems en ocho síndromes. Cinco de estas ocho categorías se agrupan en dos dimensiones más amplias: problemas internalizantes (ansioso/depresivo, introvertido/depresivo y quejas somáticas) y problemas externalizantes (infringir las normas y conducta agresiva).

Los resultados indican que tanto el afecto como la disciplina inductiva de los padres constituyen prácticas positivas que correlacionan negativamente con algunos de los problemas analizados. Por el contrario, las prácticas negativas, tales como el rechazo y la disciplina rígida tanto del padre como de la madre, se relacionan positivamente con la mayoría de los problemas analizados.

Palabras clave: prácticas educativas, problemas de ajuste, alumnos universitarios

\section{ABSTRACT}

This study aims to analyze the relationships between affection / rejection and the types of discipline that show both the father and the mother and the internalizing and externalizing problems reported by university students.

The Escala de Afecto «EA» and the Escala de Normas y Exigencias «ENE» (Fuentes, Motrico and Bersabé, 1999; Bersabé, Fuentes and Motrico, 2001) were applied in their version for children (EA- 
$\mathrm{H}$ and ENE-H). We also use the Youth Self-Report (Achenbach, 1991) by the proposal of Ivanova et al. (2007), who group the items into eight síndromes. Five of these eight categories are grouped into two dimensions: internalizing problems (anxious / depressed, introverted / depressed and somatic complaints) and externalizing problems (delinquent behavior and aggressive behavior).

The results indicate that both affection and the inductive discipline of parents are positive practices that correlate negatively with some of the problems analyzed. On the contrary, negative practices, such as rejection and rigid discipline of both father and mother, are positively related to most of the problems analyzed.

Key words: educational practices, adjustment problems, college students

\section{INTRODUCCIÓN}

Las relaciones que mantienen los adolescentes con sus padres es un factor determinante que influye en su ajuste psicológico. En los últimos años son numerosos los trabajos que podemos encontrar que muestran como la calidad de las relaciones entre padres e hijos influye en el ajuste psicosocial de éstos (García, Cerezo, De la Torre, Carpio y Casanova, 2011, Nishikawa, Sundbom y Hägglöf, 2010; Kapi, Veltsista, Kavadias, Lekea, Bakoula, 2007; Torrente y Vazsonyi, 2008).

Tradicionalmente han existido dos aproximaciones al análisis de la actuación de los padres, una aproximación tipológica, representada por la propuesta de diferentes estilos educativos y una aproximación dimensional que se centra en las características conductuales de los padres.

La aproximación tipológica (Baumrind, 1971; Maccoby y Martin, 1983; Steinberg, Lamborn, Darling, Mounts y Dornbusch, 1994) propone diferentes estilos educativos (padres democráticos, padres negligentes, padres permisivos y padres autoritarios) obtenidos de la combinación de las dimensiones de afecto y control de los padres (Baumrind, 1996; Maccoby y Martin, 1983). Recientemente se han comenzado a señalar limitaciones en la aproximación tipológica, por ejemplo, el hecho de que los resultados atribuidos a los estilos de padres varían en función de características culturales y étnicas (Bugental y Grusec, 2006; Chao, 2001; Musitu y García, 2004).

Además los estilos paternos, al ser una configuración de prácticas, no permiten diferenciar las variables que influyen en el funcionamiento adolescente. Clark, Novack y Dupree (2002) señalaban que las prácticas de los padres pueden resultar más predictivas en los adolescente que los estilos. Por ello, Ato, Galián y Huescar (2007) propusieron la necesidad no tanto de dividir a los padres en categorías sino tratar de conocer como puntúan en una serie de dimensiones que se consideran continuas.

La aproximación dimensional, por tanto, atiende a las características conductuales manifestadas por los padres. Según Darling y Steinberg (1993), las prácticas de los padres ejercen influencia directa en las características de los jóvenes, mientras que las tipologías ejercen influencia indirecta. En este sentido, investigaciones recientes han centrado su trabajo en el análisis de distintas dimensiones del comportamiento paterno y materno que se relacionan con la manifestación de síntomas conductuales tanto de naturaleza internalizante como externalizante (Galambos, Barker, y Almeida, 2003; Hurtig, Taanila, Ebeling, Miettunen y Moilanen, 2005; Muris, Meesters y van den Berg, 2003; Nishikawa y colb., 2010; Rothrauff, Cooney y An, 2009; Sandoval, Lemos, y Vallejo, 2006).

Una de las principales dimensiones de la conducta de los padres analizada es el afecto. En este sentido, Lum y Phares (2005); García y colb., (2011) Oliva, Parra, Sánchez-Queija y López, (2007); Torrente y Vazsonyi (2008), señalan que el afecto es un elemento positivo en la calidad de las relaciones padres-hijos procurando mayor bienestar emocional y mejor ajuste conductual. En oposición al constructo de afecto se encuentra el de rechazo parental. La percepción de rechazo y 
ausencia de afecto son factores de riesgo que predicen la aparición de problemas de conducta tanto internalizantes como externalizantes (Akse, Hale, Engels, Raaijmakers y Meeus, 2004; Muris y colb., 2003; García y colb., 2011).

Otra característica paterna que se considera un factor de riesgo para la conducta desviada de los adolescentes ha sido el control paterno, tanto la falta de supervisión como el exceso de control mantienen una relación con la frecuencia de aparición de problemas de comportamientos antisociales y disruptivos en sus hijos (Kapi y colb., 2007, Torrente y Vazsonyi, 2008; García y colb., 2011; Oliva, Parra y Arranz, 2008). No obstante, a pesar de que los datos apoyan la importancia del control parental para la prevención de problemas de conducta (Fletcher, Steinberg y WilliamsWheeler, 2004) los investigadores no se ponen de acuerdo con respeto a los aspectos a incluir bajo esta etiqueta y los resultados no son concluyentes debido a que, como indican Eisenberg, Chang, Ma, y Huang (2009), los hallazgos difieren en función de la operacionalización de los constructos. Así, dependiendo del tipo de control los resultados pueden variar. El control de los padres democráticos (inductivo) puede proteger de los problemas, pero el control de los padres autoritarios (rígido) es un factor de riesgo.

Además la mayor parte de la investigación sobre prácticas educativas se ha centrado en las llevadas a cabo o bien por la madre, o bien por ambos padres conjuntamente, ignorando en muchas ocasiones la conducta de los padres (Silverstein, 2002). No obstante, aunque los padres mantienen relaciones menos estrechas con los adolescentes que las madres, los estudios han mostrado que su papel es significativo, de hecho se han encontrado diferencias en las valoraciones que el hijo hace sobre su relación con el padre y con la madre (Demo, Small y Savin-Williams, 1987; Rodrigo, y colb., 2004; Samper, Cortés, Mestre, Nácher y Tur, 2006). Si bien parecen existir más semejanzas que diferencias entre el funcionamiento de padres y madres (Lum y Phares, 2005) es necesario analizar las prácticas en ambos progenitores separadamente y examinar el papel del padre y de la madre, (Alonso y Román, 2005; Carpio, García, De la Torre, Cerezo y Casanova, 2014; García y colb., 2011; Lum y Phares, 2005; Milevsky, Schlechter, Netter y Keehn 2007; Ortiz, Apodaca, Etxebarria, Fuentes y López, 2008; Raya, Herruzo y Pino, 2008; Samper y colb., 2006).

En base a todo lo anterior, en este trabajo pretendemos analizar las relaciones de las dos dimensiones de la conducta de los padres (afecto y control) con las puntuaciones del YSR (Youth SelfReport, Achenbach, 1991) que representan problemas internalizantes y externalizantes en una muestra de estudiantes universitarios. La dimensión de afecto se subdivide en dos subdimensiones independientes: afecto-comunicación y crítica-rechazo. El control de los padres se mide a través de tres escalas referidas al control ejercido de forma inductiva, control ejercido de forma rígida y control ejercido de forma indulgente.

Si bien todos los trabajos hasta ahora mencionados toman como muestra a alumnos adolescentes, es conocida la importancia del entorno familiar como uno de los factores que inciden en el desarrollo del individuo a lo largo de todas las edades. Por ello, la finalidad de este trabajo es ampliar el análisis de estas relaciones a una muestra de estudiantes universitarios que pertenecen a la etapa de la adultez emergente y conocer si los padres siguen desempeñando un papel importante en el proceso de socialización de sus hijos tal y como demostró Grotevant, (1998).

\section{MÉTODO}

\section{Participantes}

En este estudio participaron 123 estudiantes de primer curso de diferentes Grados, todos ellos impartidos en la Facultad de Humanidades y Ciencias de la Educación de la Universidad de Jaén. Los estudiantes cursaban los Grados de Historia del Arte, Estudios Ingleses, Psicología, Educación 


\section{PRÁCTICAS EDUCATIVAS DE LOS PADRES Y PROBLEMAS DE AJUSTE EN ESTUDIANTES UNIVERSITARIOS}

Infantil y Educación Primaria. Todos cumplían el criterios de tener 18 años en el momento de la pasación.

La aplicación de las pruebas tuvo lugar durante una sesión de una hora de duración, en horario de clase de los distintos grados (historia del arte, estudios ingleses, psicología, educación infantil y educación primaria) y en presencia del profesor y los investigadores. Se pidió la colaboración del alumnado y se les garantizó que la respuesta a los cuestionarios era totalmente voluntaria y confidencial.

\section{Instrumentos}

Escala de Afecto (EA) (Fuentes, Motrico y Bersabé, 1999; Bersabé, Fuentes, y Motrico, 2001). Se compone de dos factores 1) afecto-comunicación ("Puedo contar con él/ella cuando lo necesito") y 2) crítica-rechazo de los padres hacia sus hijos ("Le gustaría que fuera diferente"). Cada uno de los factores consta de 10 ítems que se contestan en una escala tipo Likert con 5 grados de frecuencia $(1=$ nunca, 2 = pocas veces, $3=$ algunas veces, $4=$ a menudo y $5=$ siempre $)$. La puntuación total de cada factor está comprendida entre 10 y 50.

Escala de Normas y Exigencias (ENE) (Fuentes, Motrico y Bersabé, 1999; Bersabé, Fuentes, y Motrico, 2001). Consta de 3 factores: 1) forma inductiva ("Me anima a hacer las cosas por mí mismo/a"), 2) forma rígida ("Intenta controlar mi vida en todo momento") y 3) forma indulgente ("Me da libertad total para que haga lo que quiera). Estos tres factores evalúan la forma que tienen los padres de establecer y exigir el cumplimiento de las normas. Los dos primeros factores tienen 10 ítems, y el tercero se compone de 8. La escala de respuesta es la misma que en la EA, por lo que la puntuación total de los dos primeros factores también está comprendida entre 10 y 50. Únicamente la puntuación del tercer factor varía entre 8 y 40.

Ambas escalas se presentan en dos versiones: una para ser contestada por los hijos (EA-H y ENE-H) y otra por sus padres (EA-P y ENE-H). En la versión de los hijos, el adolescente debe responder al contenido de cada ítem según la percepción que tiene del estilo educativo de su padre y de su madre ("Antes de castigarme escucha mis razones"). En la versión de los padres, éstos contestan los ítems refiriéndose a cómo es su conducta concreta con su hijo/a ("Antes de castigarle escucho sus razones"). En este trabajo utilizamos solo la versión para hijos.

YSR (Youth Self- Report) Achenbach (1991). El Youth Self-Report (YSR), es una medida de autoinforme elaborada para evaluar las competencias psicosociales así como distintos problemas de naturaleza conductual y emocional en niños y adolescentes de edades comprendidas entre los 11 y los 18 años de edad. La primera parte de esta prueba valora las capacidades deportivas, sociales y académicas de niños y adolescentes. La segunda parte, compuesta por 112 ítems, de los cuales 16 examinan la frecuencia de aparición de conductas adaptativas o prosociales, se circunscribe a la evaluación de un extenso conjunto de conductas problemáticas. El formato de respuesta requiere del participante la emisión de un juicio relativo al grado mediante el cual cada afirmación se corresponde con el comportamiento exhibido durante los últimos seis meses, siendo tres las opciones de respuesta $(0$ = la afirmación no es cierta 0 no es aplicable a mi persona, 1 = la afirmación es aplicable a mi persona en algunas ocasiones, 2 = la afirmación se ajusta muy a menudo o frecuentemente a mi persona). Una mayor puntuación se corresponde con una manifestación más elevada de problemas emocionales y comportamentales.

EI YSR ha sido objeto de adaptación, estudio y empleo en muestras de adolescentes españoles (Lemos, Vallejo y Sandoval, 2002; Sandoval, Lemos y Vallejo, 2006; Zubeidat, Fernández-Parra, Ortega, Vallejo y Sierra, 2009). En este estudio se ha optado por utilizar los resultados obtenidos por Ivanova, Acchenbach, Rescolar, Dumenci, Almqvist, Bilenberg et al., (2007) quienes mediante aná- 
lisis factorial confirmatorio agruparon 89 ítems en ocho síndromes relacionados entre sí: ansioso/depresivo, introvertido/depresivo, quejas somáticas, problemas sociales, problemas de pensamiento problemas de atención, infringir las normas y conducta agresiva, Tres de esas ocho categorías estaban referidos a síntomas internalizantes (ansioso/depresivo, introvertido/depresivo y quejas somáticas) y síntomas externalizantes (infringir las normas y conducta agresiva)

\section{RESULTADOS}

El objetivo del presente estudio fue determinar la relación existente entre las prácticas educativas paternas y la presencia de problemas de conducta tanto internalizantes como externalizantes en estudiantes universitarios.

Para ello se llevó a cabo un análisis de correlación de Pearson. Como podemos ver en la tabla 1 la dimensión de afecto tanto del padre como de la madre presenta correlación negativa y significativa con la mayoría de los problemas que informan los estudiantes universitarios: agresividad, problemas de pensamiento, infringir reglas, problemas de atención y problemas sociales en el caso de las madres. Con respecto al rechazo/crítica que ejerce tanto el padre como la madre correlaciona de forma positiva y significativa con todos los problemas que informan los adolescentes: agresividad, ansiedad/depresión, problemas de pensamiento, infringir reglas, problemas sociales, problemas de atención y quejas somáticas en el caso de la madre.

Tabla 1. Correlaciones entre prácticas educativas paternas y problemas de conductas del YSR (Youth Self- Report, Achenbach, 1991).

\begin{tabular}{|c|c|c|c|c|c|c|c|c|c|c|}
\hline & \multicolumn{2}{|c|}{ Afecto } & \multicolumn{2}{|c|}{ Rechazo } & \multicolumn{2}{|c|}{$\begin{array}{l}\text { Disciplina } \\
\text { Inductiva }\end{array}$} & \multicolumn{2}{|c|}{$\begin{array}{l}\text { Disciplina } \\
\text { Indulgente }\end{array}$} & \multicolumn{2}{|c|}{$\begin{array}{c}\text { Disciplina } \\
\text { Rígida }\end{array}$} \\
\hline & padre & madre & padre & madre & Padre & madre & Padre & madre & Padre & madre \\
\hline Agresividad &,$- 214^{*}$ &,$- 262^{* *}$ &, $400^{* *}$ &, $466^{* *}$ &,$- 182^{*}$ &,- 172 &,- 026 &,- 022 &, $380^{* *}$ &, $303^{* *}$ \\
\hline Ansioso/depr &,- 168 &,- 131 &, $210^{*}$ &, $194^{*}$ &,- 139 &,- 150 & ,069 &, 059 &, $241^{* *}$ &, $262^{* *}$ \\
\hline Prob. pensam &,$- 186^{*}$ &,$- 276^{* *}$ &, $254^{* *}$ &, $273^{* *}$ &,$- 200^{*}$ &,$- 186^{*}$ & ,037 & ,088 & ,153 &, 083 \\
\hline Infringir reglas &,$- 305^{* *}$ &,$- 374^{* *}$ &, $257^{* *}$ &, $343^{* *}$ &,$- 214^{*}$ &,$- 223^{*}$ & , 169 &,- 056 &, 144 &, $305^{* *}$ \\
\hline Prob sociales &,- 135 &,$- 256^{* *}$ &, $312^{* *}$ &, $314^{* *}$ &,- 169 &,- 128 &,- 043 &,- 037 &, $261^{* *}$ &, $280^{* *}$ \\
\hline Quejas somat &,- 156 &,- 121 &, 142 &, $179^{*}$ &,- 164 &,- 096 &,- 011 & 039 &, $209^{*}$ &, 177 \\
\hline Prob atención &,$- 280^{* *}$ &,$- 268^{* *}$ &, $372^{* *}$ &, $353^{* *}$ &,$- 265^{* *}$ &,$- 237^{* *}$ & ,028 &,- 062 &, $295^{* *}$ &, $312^{* *}$ \\
\hline Introv/depres &,- 086 &,- 163 & ,144 &, 169 &,- 116 &,- 100 &,- 047 & ,017 &, $255^{* *}$ &, $223^{*}$ \\
\hline
\end{tabular}

${ }^{*} \mathrm{p}<0,01 ; * \mathrm{p}<0,001$

La disciplina inductiva que ejercen tanto los padres como las madres también mantiene correlaciones negativas significativas con los problemas de pensamiento, infringir las reglas y problemas de atención. En el caso de los padres también presenta correlaciones negativas en relación a la agresividad.

Con respecto la disciplina rígida de la padres y madres mantiene relaciones positivas y significativas con todas y cada una de las dimensiones de problemas informados por los adolecentes: agresividad, ansiedad/depresión, problemas sociales, problemas de atención e introversión/depre- 


\section{PRÁCTICAS EDUCATIVAS DE LOS PADRES Y PROBLEMAS DE AJUSTE EN ESTUDIANTES UNIVERSITARIOS}

sión. Además, las quejas somáticas en el caso de los padres e infringir las reglas en el caso de las madres.

En segundo lugar se analizaron las correlaciones entre las prácticas educativas paternas y los problemas internalizantes y externalizantes siguiendo la clasificación de Ivanova y colb., (2007) quienes agrupan los ocho síndromes del YSR en dos dimensiones más amplias que recogen cinco de esos ocho síndromes: Problemas internalizantes (ansioso/depresivo, introvertido/depresivo y quejas somáticas) y problemas externalizantes (infringir las normas y conducta agresiva).

Tal y como podemos ver en la tabla 2, la percepción de afecto y la disciplina inductiva, prácticas que se pueden considerar como positivas se relacionan negativamente con la manifestación de problemas externalizantes. Por el contrario la percepción de rechazo así como la disciplina rígida, prácticas que se pueden considerar como negativas, se relacionan positivamente con los problemas internalizantes y externalizantes.

Tabla 2. Correlaciones entre prácticas educativas paternas y problemas internalizantes y externalizantes en adolescentes

\begin{tabular}{|c|c|c|c|c|c|c|c|c|c|c|}
\hline & \multicolumn{2}{|c|}{ Afecto } & \multicolumn{2}{|c|}{ Rechazo } & \multicolumn{2}{|c|}{$\begin{array}{l}\text { Disciplina } \\
\text { Inductiva }\end{array}$} & \multicolumn{2}{|c|}{$\begin{array}{l}\text { Disciplina } \\
\text { Indulgente }\end{array}$} & \multicolumn{2}{|c|}{$\begin{array}{l}\text { Disciplina } \\
\text { Rígida }\end{array}$} \\
\hline & padre & madre & padre & madre & padre & madre & padre & madre & padre & madre \\
\hline INTERNALI &,- 161 &,- 168 & $198^{*}$ &, $217^{*}$ &,- 166 &,- 138 &, 001 &, 045 &, $285^{* *}$ &, $266^{* *}$ \\
\hline EXTERNALI &,$- 291^{* *}$ &,$- 357^{* *}$ &, $378^{* *}$ &, $464^{* *}$ &,$- 224^{*}$ &,$- 223^{*}$ &, 074 &,- 043 &, $306^{* *}$ &, $345^{* *}$ \\
\hline
\end{tabular}

\section{DISCUSIÓN}

En este trabajo se han analizado las relaciones entre las prácticas educativas de los padres y las madres y la manifestación de problemas internalizantes y externalizantes en estudiantes universitarios españoles.

En el análisis correlacional realizado podemos observar que el afecto y la disciplina inductiva correlacionan negativamente con los problemas comportamentales que presentan sus hijos, por lo que pueden considerarse ambas dimensiones como factores protectores para los estudiantes universitarios. En el caso del rechazo o la disciplina rígida las correlaciones positivas con los problemas de conducta es un indicador del posible factor de riesgo para los estudiantes universitarios. Estos resultados coinciden con los obtenidos en otras investigaciones como Carpio y colb., (2014); García y colb. (2011); Oliva y colb., (2007).

Con respecto al afecto, dimensión que podemos considerar como positiva, las relaciones son significativas con la mayoría de las dimensiones comportamentales tanto del padre como de la madres excepto en las dimensiones de ansiedad y depresión, quejas somáticas, introversión y depresión, así como los problemas sociales del padre. Los padres y madres percibidos por sus hijos como afectuosos presentaban menores problemas relacionados con la agresividad, infringir reglas o normas, problemas de pensamiento y problemas de atención, al mismo tiempo los hijos de las madres percibidas como afectuosas presentaban menos problemas sociales. Atendiendo a la clasificación llevada a cabo por Ivanova y colb., (2007) la percepción de afecto por parte de los estudiantes universitarios se relaciona negativamente con la aparición de problemas externalizantes. Estos datos apoyan la idea de que el afecto es un factor protector que actúa logrando una mejor calidad de las relaciones entre padres e hijos estudiantes universitarios y evita la aparición de pro- 
blemas comportamentales, datos que coinciden con estudios previos realizados por Carpio y colb., (2014); García y colb. (2011); Lum y Phares (2005); Oliva y colb., (2007) y Torrente y Vazsonyi (2008).

En el caso del rechazo parental, dimensión que podemos considerar como negativa, las relaciones son significativas con todas las dimensiones excepto los problemas de introversión /depresión y las quejas somáticas en el caso de los padres. Los estudiantes universitarios que perciben en sus padres distancia y rechazo están más predispuestos a desarrollar comportamientos agresivos, problemas sociales, transgresión de normas, así como problemas sociales, problemas atencionales, problemas de pensamiento y ansiedad. En general, la percepción de rechazo y ausencia de afecto son factores de riesgo que predicen la aparición de problemas de conducta tanto internalizantes como externalizantes (Akse y colb., 2004; Muris y colb., 2003 García y colb., 2011).

En relación a la disciplina paterna, nuestros datos indican como el tipo de disciplina determina resultados diferentes en el desarrollo de problemas de conducta. Los padres y madres que utilizan una disciplina inductiva, previenen la aparición de problemas relacionados con la transgresión de normas, problemas de pensamiento y problemas de atención. En el caso de los padres también previenen la aparición de conductas agresivas. Los padres y madres que utilizan una disciplina rígida con sus hijos estudiantes universitarios, éstos informan de conductas agresivas, problemas atencionales, problemas sociales, ansiedad/depresión e introversión /agresión. Además, en el caso de la disciplina rígida en los padres también informan de quejas somáticas y en el caso de as madres de transgresión de normas. En definitiva, la disciplina inductiva protege a los estudiantes universitarios del desarrollo problemas externalizantes, sin embargo la disciplina rígida en un factor de riesgo tanto para problemas internalizantes como problemas externalizantes, datos que coinciden con las conclusiones de otros estudios como García y colb., (2014); Prevatt, (2003) y Ramírez (2002) que señalaban que las prácticas negativas más que las positivas son las que predicen los problemas de conducta en los niños.

En cuanto a la comparación entre las prácticas educativas del padre y de la madre encontramos algunas diferencias en la percepción que presentan de ambos progenitores. Así, las correlaciones de las madres en comparación con los padres, son ligeramente más altas en afecto y en rechazo en algunas dimensiones, sin embargo no hay patrón que pueda ser concluyente. Estos resultados van en la línea de los trabajos de Nishikawa y colb., (2010) que concluyeron que la conducta de los padres hacia los adolecentes es tan importante como la de las madres para conseguir el mejor bienestar de sus hijos.

En definitiva, de este trabajo podemos extraer una interesante conclusión relacionada con la idea de procurar prácticas educativas positivas y evitar prácticas educativas negativas. Las prácticas educativas negativas resultan ser más predictivas de la aparición de problemas de conductas externalizantes e internalizantes que las prácticas educativas positivas (García y colb. 2011). Estos resultados tienen importantes implicaciones didácticas relacionados con el desarrollo de programas de intervención y principalmente de prevención destinados a mostrar a los padres la importancia de las prácticas educativas para evitar la aparición de problemas conductuales y emocionales en sus hijos adolescentes.

Para finalizar hay que señalar algunas limitaciones del presente estudio. La primera se refiere al empleo de autoinformes para recabar la información de interés. En este sentido se confía en la sinceridad de los participantes a los que se garantizaba el anonimato de sus respuestas y así evitar las respuestas socialmente deseables. En segundo lugar, el carácter transversal de la investigación impide explorar la bidireccionalidad de los resultados obtenidos. Este hecho resulta especialmente interesante porque puede ocurrir que las características emocionales de los hijos también influyan en el estilo adoptado por los padres. 


\section{PRÁCTICAS EDUCATIVAS DE LOS PADRES Y PROBLEMAS DE AJUSTE EN ESTUDIANTES UNIVERSITARIOS}

\section{REFERENCIAS BIBLIOGRÁFICAS}

Achenbach, T.M. (1991). Manual for the Youth Self-Report and 1991 YSR profile. Burlington,V.T: University of Vermont.

Akse, J., Hale III, W.W., Engels, R. C.M.E., Raaijmakers, Q.A.W. y Meeus, W.H.J. (2004). Personality, perceived parental rejection and problems behavior in adolescence. Social Psychiatry Epidemiology, 39, 980-988.

Alonso, J. y Román, J.M. (2005). Prácticas educativas familiares y autoestima. Psicothema, 17, 7682

Ato, E., Galián, M. D. y Huescar, E. (2007). Relaciones entre estilos educativos, temperamento y ajuste social en la infancia. Una revisión. Anales de Psicología, 23, 33-40.

Baumrind, D. (1971). Current patterns of parental authority. Developmental Psychology, 4(1), 1-103. doi: 10.1037/h0030372.

Bersabé, R., Fuentes, M. J. y Motrico, E. (2001). Análisis psicométricos de dos escalas para evaluar estilos educativos parentales. Psicothema, 13, 678-684.

Bugental, D.B., \& Grusec, J.E. (2006).Socialization processes. In W. Damon \& R. Lerner (Eds.), Handbook of child psychology (6th ed.). New York: Wiley.

Carpio-Fernández, M.V., García, M.C., De la Torre, M.J., Cerezo, M.T. y Casanova, P.F. (2014). Consistencia e inconsistencia en los estilos de madres y padres y ajuste psicológico en los adolescentes, Revista de Psicología. International Journal of Developmental and Educational Psychology, 1(2), 447-456. ISNN:0214-9877

Chao, R.K. (2001). Extending research on the consequences of parenting style for Chinese Americans and European Americans. Child Development, 72, 1.832-1.84

Clark, R., Novack, J.D. y Dupree, D. (2002). Relationship of perceived parenting practices to anger regulation and coping strategies in african-american adolescents. Journal of Adolescence, 25, 373-384.

Darling, N. y Steinberg, L. (1993). Parenting style as context: An integrative model. Psychological Bulletin, 113, 487-496.

Demo, D.H.; Small, S. A. y Savin-Williams, R.C. (1987). Family relations and the self-esteem of adolescents and their parents. Journal of Marriage and the Family, 49, 705-715

Eisenberg, N., Chang, L., Ma, Y. y Huang, X. (2009). Relations of parenting style to Chinese children s effortful control, ego resilience, and maladjustment. Development and psychopathology, 21, 455-477

Fletcher, A. C., Steinberg, L., y Sellers, E. B. (1999). Adolescents' well-being as a function of perceived interparental consistency. Journal of Marriage and the Family, 61(3), 599-610.

Fuentes, M. J., Motrico, E., y Bersabé, R. M. (1999). Escala de afecto (EA) y Escala de normas y exigencias (ENE): versión hijos y versión padres. Universidad de Málaga.

Galambos, N.L., Barker, E.T., y Almeida, D.M. (2003). Parents do matter: Trajectories of change in externalizing and internalizing problems in early adolescence. Child Development, 74, 578-594.

García-Linares, M. C., Cerezo, M. T., De la Torre, M. J., Carpio, M. V., y Casanova, P. F. (2011). Prácticas educativas paternas y problemas internalizantes y externalizantes en adolescentes españoles. Psicothema, 23, 654-659

Grotevant, H. D. (1998). Adolescent development in family contexts. In N. Eisenberg (Vol. Ed.) \& W. Damon (Series Ed.), Handbook of child psychology: Social, emotional, and personality development (Vol. 3, pp. 1097-1149). New York: Wiley.

Hurtig T, Taanila A, Ebeling H, Miettunen J, Moilanen I. (2005) Attention and behavioural problems of Finnish adolescents may be related to family environment. European Child \& Adolescent Psychiatry, 14, 471-8. 
Ivanova, M.Y., Achenbach, T.M., Rescorla, L.A., Dumenci, L., Almqvist, F., Bilenberg, N., et al. (2007). The Generalizability of the Youth Self-Report Syndrome Structure in 23 Societies. Journal of Consulting and Clinical Psychology, 75, 729-738

Kapi, A., Veltsista, A., Kavadias, G., Lekea, V. y Bakoula, C. (2007). Social determinants of selfreported emotional and Behavioral problems in Greek adolescents. Social Psychiatry and Psychiatry Epidemiology, 42, 594-598.

Lemos, S., Vallejo, G., y Sandoval, M. (2002). Estructura factorial del Youth Self-Report (YSR). Psicothema, 14, 816-822.

Lum, J.J. y Phares, V. (2005). Assessing the emotional availabitity of parents. Journal of Psychopathology and Behavioral Assessment, 27, 211-226.

Maccoby, E.E. y Martin, J.(1983). Socialization in the context of the family. Parent-child interation. In P.H. Mussen y E.M. Hetherington Handbook of child psychology. Vol. 4 Socialization, personality and de social development (4th $e d$. Pp 1-102) New York: Wiley.

Milevsky, A., Schlechter, M, Netter, S. y Keehn, D. (2007). Maternal and paternal parenting styles in adolescents: Associations with self-esteem, depression and life-satisfaction. Journal of Child and Family Studies, 16, 39-47.

Muris, P., Meesters, C.M.G. y van den Berg, S. (2003). Internalizing and externalilzing problems as correlates of self-reported attachement style and perceivec practices rearing in normal adolescents. Journal of Child and Family Studies, 12, 171-183.

Musitu, G. y Garcia, J.F. (2004) Consecuencias de la socialización familiar en la cultura española. Psicothema, 16, 288-293

Nishikawa, S.; Sundbom. E. y Hägglöf, B. (2010) Influence of perceived parental rearing on adolescent self-concept and internalizing and externalizing problems in Japan. Journal of Child and Family Studies, 19, 57-66

Oliva, A., Parra, A. y Arranz, E. (2008). Estilos relacionales parentales y ajuste adolescente. Infancia y Aprendizaje, 31, 93-106

Oliva, A., Parra, A., Sánchez-Queija, I. y López. F. (2007). Estilos educativos materno y paterno: Evaluación y relación con el ajuste adolescente. Anales de Psicología, 23 (1), 49-56.

Ortiz, M.J.; Apodaca, P., Etxebarria I., Fuentes, M.J. y López, F. (2008). Predictores familiares de la internalización moral en la infancia. Psicothema, 20, 712-717.

Prevatt, F.F. (2003). The contribution of parenting practices in a risk and resiliency model of children s adjustment. British Journal of Developmental Psychology, 21, 469-480.

Ramírez, M.A. (2002) Prácticas de crianza de riesgo y problemas de conducta en los hijos. Apuntes de Psicología, 20, 273-282

Raya, A. F.; Herruzo, J. y Pino, M.J. (2008). El estilo de crianza parental y su relación con la hiperactividad. Psicothema, 20, 691-696.

Rodrigo, Mํㅡㄹ. J., Máiquez, Mํㅡㄴ. García, M., Mendoza, R., Rubio, A., Martínez, A. y Martín, J.C. (2004). Relaciones padres-hijos y estilos de vida en la adolescencia. Psicothema, 16 203-210.

Rothrauff, T., Cooney, T., \& An, J. S. (2009). Remembered parenting styles and adjustment in middle and late adulthood. Journal of Gerontology: Social Sciences, 64, 137-146

Samper, P., Cortés, M.T., Mestre, V., Nácher, M.J. y Tur, A.M. (2006). Adaptación del Child's Report of Parent Behavior Inventory a población española. Psicothema, 18, 263-271.

Sandoval, M., Lemos, S., y Vallejo, G. (2006). Self-reported competentes and problems in Spanish adolescents: A normative study of the YSR. Psicothema, 18(4), 804-809.

Silverstein, L.B. (2002). Fathers and families. In J.P. McHale y W.S. Grolnick (Eds.) Retrospect and prospect in the psychological study of families (pp. 35-64). Mahwah, NJ: Erlbaum. 


\section{PRÁCTICAS EDUCATIVAS DE LOS PADRES Y PROBLEMAS DE AJUSTE EN ESTUDIANTES UNIVERSITARIOS}

Steinberg, L., Lamborn, S.D., Darling, N., Mounts, N.S. y Dornbusch, S.M. (1994).Overtime changes in adjustment and competence among adolescents from authoritative, authoritarian, indulgent and neglectful families. Child Development, 65, 754-770.

Torrente, G. y Vazsonyi, A.T. (2008). The salience of the family in antisocial and delinquent behavior among spanish adolescents. The Journal of Genetic Psychology, 169, 187-197

Zubeidat,I, Fernández-Parra,A, Ortega, J.M., Vallejo, M.A., y Sierra, J.C. (2009). Características psicosociales y psicopatológicas en una muestra de adolescentes españoles a partir de youth self.report /11-18, Anales de Psicología,25, (1) 60-69. 